\title{
Abdominal-wall Endometriosis Occurring at the Wound Site after a Caesarian Section
}

\author{
Ryuta Taniguchi, Noritaka Minagawa, Koji Onitsuka and Yoshitaka Sakamoto \\ Department of Surgery, Kyusyu-Rosai Hospital Moji Medical Center
}

\begin{abstract}
A 47-year-old woman underwent caesarian section at the age of 23 and 33 . Subsequently, she underwent another operation for an umbilical hernia and abdominal-wall tumor of umbilicus when she was 39 years old. Abdominal-wall endometriosis of umbilicus was diagnosed based on the results of pathological examination. There was no post-operative recurrence. At the age of 44 years, two abdominalwall tumors with cyclical lower abdominal pain were observed in the lower abdominal region. She presented with pain at our hospital and had a palpable abdominal-wall tumor along the caesarian section scar. Tumors were another legions at the age of 39 years. Contrast-enhanced computed tomography revealed that the two tumors had invaded the rectus abdominis muscle. Both tumors were $35 \mathrm{~mm}$ in size. Magnetic resonance imaging revealed blood component into tumors. We suspected abdominalwall endometriosis based on these findings. Coreneedle biopsy was not performed due to the risk of re-implantation and dissemination. Surgical excision was used to remove the tumors along with some surrounding tissues. Pathological analysis revealed that the tumors consisted of endometriosis with glands and endometrial stroma. Approximately $79 \%$ of abdominal-wall endometriosis occurs in the operative scar after a caesarian section. As there are no treatment guidelines, wide excision with normal tissue surrounding the tumor is recommended.
\end{abstract}

Received: October 3, 2018/ Accepted: November 10, 2018 Correspondence to: Ryuta Taniguchi

Department of Surgery, Kyusyu-Rosai Moji Hospital 3- 1, Higasiminatomachi, Moji, Kitakyusyu, Fukuoka 801-8502, Japan
Key words: heterochrony, abdominal wall endometriosis, caesarian section

\section{Introduction}

Endometriosis is a common gynecological disease that occurs in patients during their reproductive age $^{1)}$. Endometriosis is characterized by ectopic glands and stroma that occurred from the outside the uterine cavity, such as the extremities, central nervous system, lungs, pleurae and liver. Most cases of endometriosis occur within the pelvic cavity, such as the ovaries, pouch of Douglas, sacrouterine ligament, vesicovaginal septum, and the uterine cervix. The incidence of an abdominal-wall endometriosis (AWE) ranges from 0.03 to $3.5 \%$ of all endometriosis cases ${ }^{1)}$. The incidence of AWE is extremely rare. In this case report, we describe a patient who was diagnosed with AWE and who previously underwent heterochrony AWE.

\section{Case report}

A 47-year-old woman (gravida 2, para 2) presented with lower abdominal pain and masses. This patient had undergone caesarian section at the ages of 23 and 33. She also underwent an operation for umbilical hernia and abdominal wall tumor of umbilicus at the age of 39. The pathological findings observed a diagnosis of $\mathrm{AWE}$ and revealed proliferation of endometrioid glands with endometrioid stroma and hemorrhage. Postoperatively, abdominal pain and palpable tumors were absent. Five years later, she had two tumors accompanied by cyclical lower abdominal pain since she was 44 years old; thus, she underwent a laparoscopic hysterectomy at another hospital because of a hysteromyoma at the age of 46 . Two abdominal tumors were unrecognized at this time. As a result, hys- 


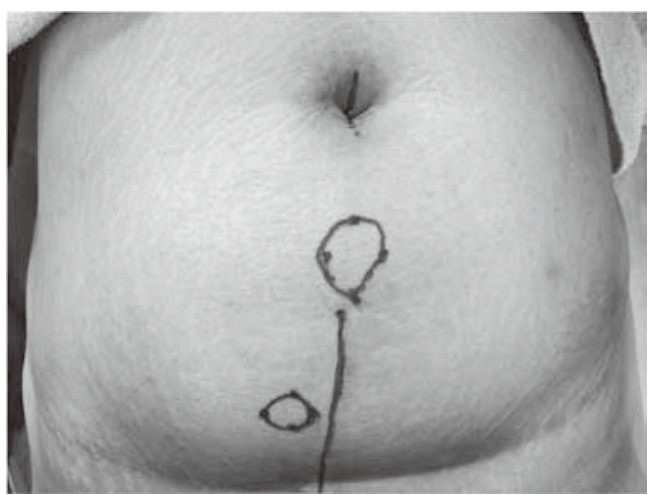

Fig. 1 Physical examination revealed palpable tumors at the upper and right side of a caesarian section scar with tenderness.

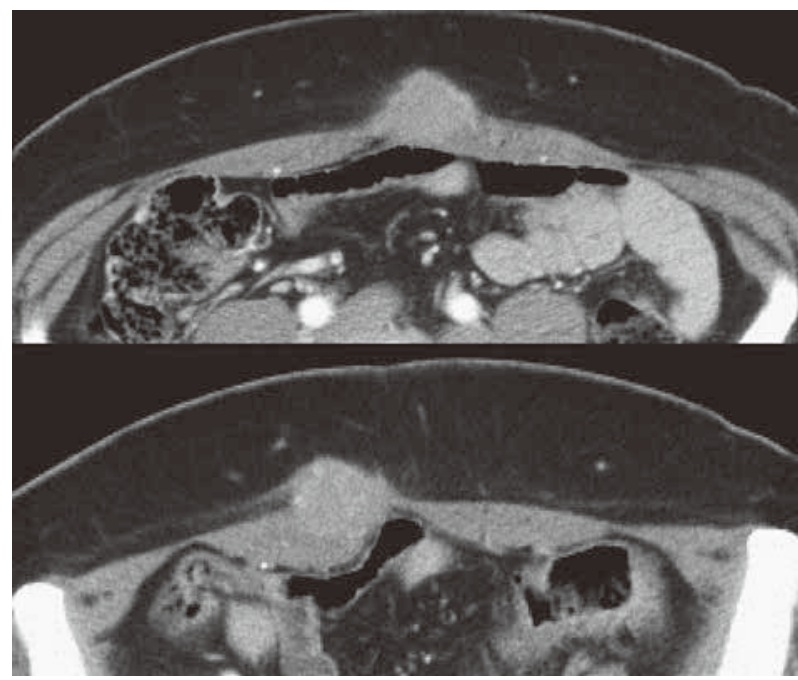

Fig. 2 a, b: Abdominal contrast-enhanced CT revealed two of invasive tumors that were approximately $35 \mathrm{~mm}$ in size each in the rectus abdominis muscle. We noted an enhanced effect and no necrosis.

teromyoma was not a cause of pain. Because her lower abdominal pain did not improve, she visited our hospital. Physical examination showed palpable tumors at the upper and right side of the caesarian section scar with tenderness (Fig. 1). Laboratory results included the following: Luteinizing hormone, LH $9.35 \mathrm{mIU} / \mathrm{mL}$; Follicle stimulating hormone, FSH $27.31 \mathrm{mIU} / \mathrm{mL}$; Prolactin, $9.15 \mathrm{ng} / \mathrm{mL}$; E2, $20 \mathrm{pg} / \mathrm{mL}$ within normal limits; carbohydrate antigen 125, 109 $\mathrm{U} / \mathrm{mL}$. Abdominal contrast-enhanced computed tomography (CT) revealed two invasive tumors that were both approximately $35 \mathrm{~mm}$ in size in the rectus abdominis muscle. There were enhanced effects and no necrosis (Fig. 2a, b). Magnetic resonance imaging (MRI) revealed similar findings and blood component into tumors (Fig. 3a, b). Due to these findings, AWE was suspected. This case revealed a heterochronic AWE after caesarian section. Since it was possible for core-needle biopsy to cause dissemination and implantation of endometrial tissues, complete resection of the tumors was performed without biopsy. The surgical excision included 10 


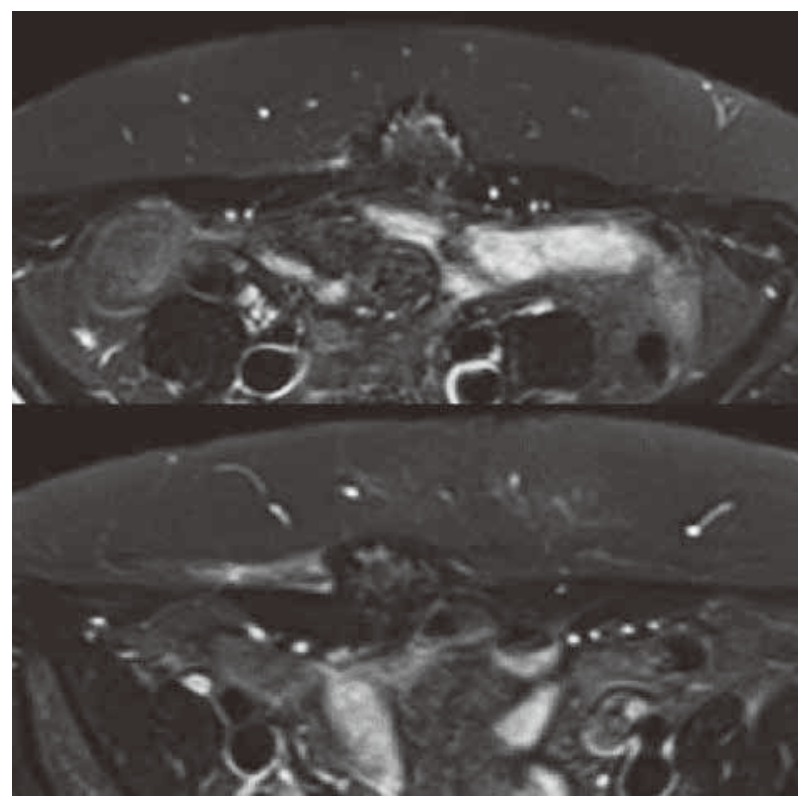

Fig. 3 a, b: MRI revealed the similar findings and a blood component.

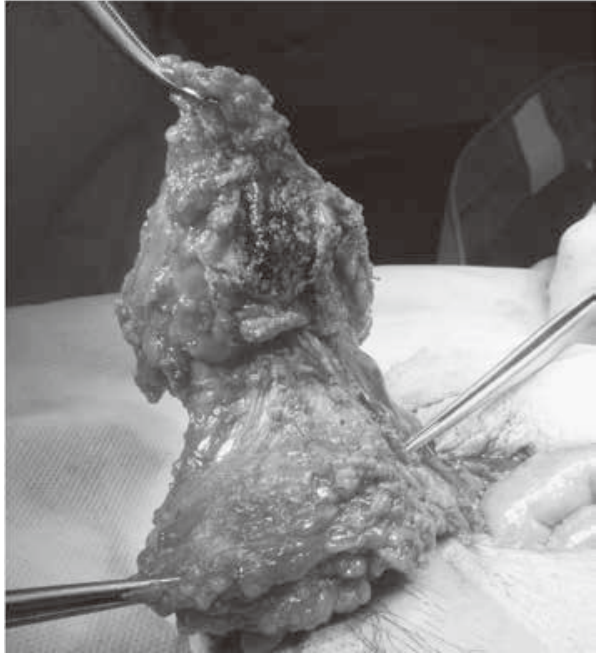

Fig. 4 Complete resection of tumors was performed. The surgical excision included $10 \mathrm{~mm}$ of the surrounding normal tissues with the tumors to prevent recurrence.

$\mathrm{mm}$ of the surrounding normal tissue with tumors to prevent recurrence (Fig. 4). A mesh reconstruction was not necessary. Macroscopically, the tumors were light gray and $35 \mathrm{~mm}$ in size each. Pathological testing revealed that these tumors consisted of endometriosis with glands and endometrial stroma. $\frac{a}{b}$

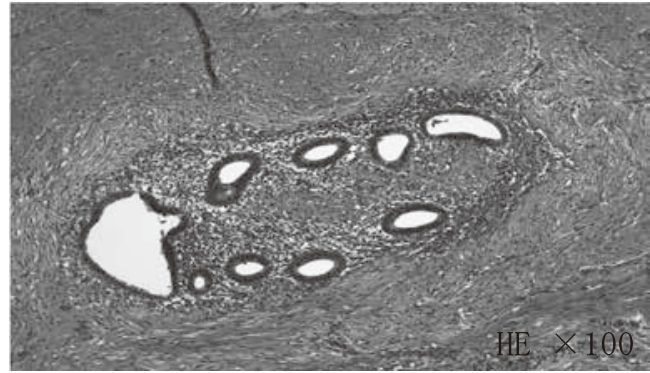

Fig. 5 These tumors consisted of endometriosis with glands and endometrial stroma. There was no atypical ectopic endometrium in the tissue. Furthermore, we noted an expanded duct, bleeding, and surrounded fiberization (hematoxylin and eosin [HE] stain $\times 100$ ).

There was no atypical ectopic endometrium in the tissues. Furthermore, there were expanded ducts, bleeding, and surrounding fiberization (Fig. 5) and the excision stump was negative. Immunostaining showed that estrogen receptor (ER) was positive, progesterone receptor ( $\mathrm{PgR})$ was positive, and cluster of differentiation (CD) 10 was positive (Fig.6a-c). Based on the findings above, we diagnosed the patient with AWE. The patient's postoperative course 

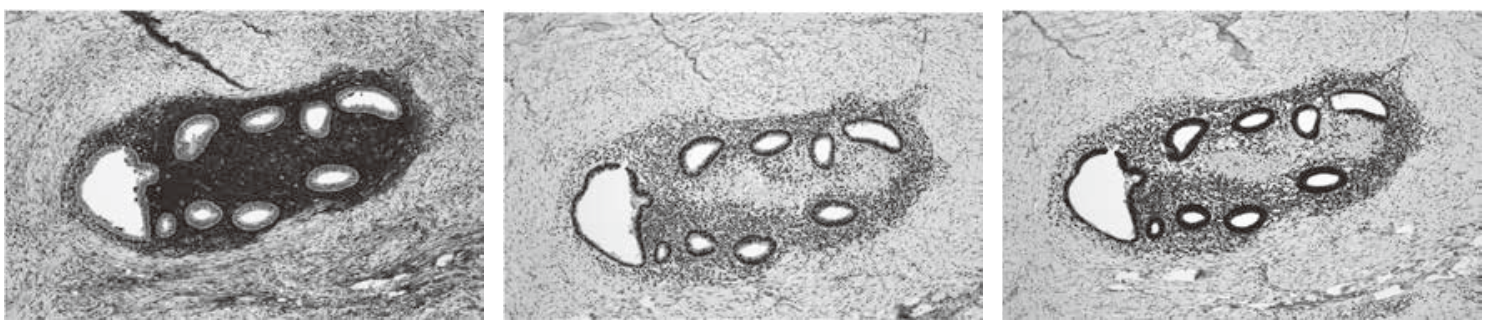

Fig. 6 a: The immunostaining showed cluster of differentiation (CD) 10 was positive (CD10 stain $\times 100$ ).

b: The immunostaining showed estrogen receptor $(E R)$ was positive (ER stain $\times 100$ ).

c: The immunostaining showed progesterone receptor $(\mathrm{PgR})$ was positive $(\mathrm{PgR}$ stain $\times 100)$.

$a|b| c$

was uneventful. There was no recurrence for oneyear.

\section{Discussion}

This patient underwent a second AWE. Since more than one year had passed, we did not consider it as a recurrence but rather the occurrence of heterochrony.

AWE that occurs in the operative wound has an estimated prevalence of $0.03-0.5 \%$ out of all types of endometriosis ${ }^{11}$. Endometriosis occurring in the operative scar after a caesarian section is estimated to be approximately $80 \%^{2}$. Skin endometriosis of surgical site endometriosis is being discussed mechanical implantation, and skin endometriosis of umbilical region is being discussed lymphatic implantation. But it is difficult for pathophysiology to explain a clear mechanism ${ }^{2}$.

Endometriosis is defined as the presence of ectopic endometrial glands and stroma outside the uterine cavity. Steck et al. reported the first cutaneous endometriosis in the world in $1966^{3)}$. At present, the cause of AWE remains unknown. AWE involves the implantation of the endometrial tissue during caesarean section at the wound site, but it was reported that AWE could occur spontaneously without uterine operation and caesarean section ${ }^{4)}$. The relative risk of AWE following caesarean sections performed before onset of labor in comparison to caesarean sections following spontaneous onset of labor was statistically higher by two-fold ${ }^{5}$. Furthermore, the caesarean section performed before 22 weeks of gestation raises the relative risk for $\mathrm{AWE}^{6)}$. This indicates that the risk of endometriosis related to the caesarean section decreases following spontaneous onset of labor, because changes in immune tolerance for the fetus are terminated ${ }^{5}$. The main symptoms of AWE are cyclical (57\%): a palpable tumor (96\%), abdominal pain (86\%), and bleeding ${ }^{7)}$. Abdominal ultrasonography (US) can confirm a cystic component and a solid component. It is possible to evaluate blood flow into the tumor, but abdominal US finding of the AWE is nonspecific. Abdominal contrast-enhanced CT can confirm the difference between sub-acute and chronic hemorrhage within the tumor. MRI may be the best modality to detect hemorrhagic lesions in endometriosis, but $\mathrm{CT}$ and MRI are also non-specific ${ }^{8)}$. AWE is often misdiagnosed such as suture granuloma, incisional hernia, primary or metastatic cancer, desmoid tumor, lymphoma, hematoma or abscess ${ }^{9)}$. Fine needle aspiration (FNA) is useful in excluding the possibility of malignancy. However, FNA is not recommended due to its association with increased risk of recurrence, dissemination, and implantation. Therefore, it is necessary to evaluate such cases carefully and on an individual basis ${ }^{10}$. The serum level of CA-125 can be slightly increased ${ }^{11)}$. It is a useful index for follow-up. Medical treatment may produce only temporary alleviation of symptoms. Symptoms recur after cessation of drugs. Since poor results with danazol, leuprolide, and progesterone were reported ${ }^{12}$, medical treatment cannot be recommended. Surgical excision is the only effective treatment and wide excision with clear margins is the key for preventing recurrence ${ }^{13)}$. Postoperative medical treatment is not effective for recurrence preven$\operatorname{tion}^{14)}$. Recurrence is rare, but AWE recurs within a year if there was an inadequate surgical resec$\operatorname{tion}^{14)}$. The risk of transformation of endometriosis to a malignant disease is currently thought to be in the range of $0.3-1.0 \%{ }^{15}$. There are three subtypes of the malignancy that can develop from AWE: endometrioid, sarcoma, and a clear cell carcinoma sub- 
type ${ }^{16)}$. Treatment of abdominal wall adenocarcinoma arising from endometriosis is not standardized. Therefore, the adoption of ovarian cancer management techniques is the standard approach ${ }^{17)}$. The prognosis is 20 months with a survival rate of 57 $\%{ }^{18)}$. In the case of our patient, abdominal wall tumor was surgically removed previously and AWE was diagnosed by pathological examination. Five years later, she presented with AWE in a different area, due to this, we diagnosed her with AWE with heterochrony onset.

\section{Conflict of Interest: None.}

\section{References}

1) Leite GK, Carvalho LF, Korkes H, et al : Scar endometrioma following obstetric surgical incisions : retrospective study on 33 cases and review of the literature. Sao Paulo Med J 127 : 270-277, 2009

2) Tsutomu M, Daisuke K, Eiichiro S, et al: A Case of Endometriosis of the Abdominal Wall Scar after Cesarean Section. Journal of Japanese College of Surgeons 34:669-673, 2009

3) Steck CW, Helwig EB:Cutaneous endometriosis. Clin Obstet Gynecol $9: 373-383,1966$

4) Ideyi SC, Schein M, Niazi M, et al : Spontaneous Endometriosis of the Abdominal Wall. Dig Surg 20 : 246-248, 2003

5) Wicherek L, Klimek M, Magierlo JS, et al : The Obstetrical History in Patients with Pfannenstiel Scar Endometriomas - An Analysis of 81 Patients. Gynecol Obstet Invest $63: 107-113,2007$

6) De Oliveria MA, de Leon AC, Freire EC, et al : Risk factors for abdominal scar endometriosis after obstetric hysterotomies: A case-control study. Acta Obstet Gynecol Scand $86: 73-80,2007$
7) Horton JD, DeZee KJ, Ahnfeldt EP, et al : Abdominal wall endometriosis : a surgeon'perspective and review of 445 cases. Am J Surg 196:207-212, 2008

8) Leu NH, Huang GS, Lu CY, et al : MRI of Endometrioma of the Abdominal Rectus Muscle : a case report. Chin J Radiol $30: 307-311,2005$

9) Wolf C, Obrist P, Ensinger C: Sonographic features of abdominal wall endometriosis. AJR 169 :916917, 1997

10) Bumpers HL, Butler KL, Best IM : Endometrioma of the abdominal wall. Am J Obstet Gynecol 187: 1709-1710, 2002

11) Luisi S, Gabbanini M, Sollazzi S, et al : Surgical scar endometriosiss after Cesarean section: A case report. Gynecol Endocrinol 22 : 284-285, 2006

12) Zhao X, Lang J, Leng J, et al : Abdominal wall endometriomas. Int J Gynecol Obstet $90: 218-222,2005$

13) Matthes G, Zabel DD, Nastala CL, et al : Endometrioma of the abdominal wall following combined abdomenasty and hysterectomy : case report and review of the literature. Ann Plast Surg 40:672-675, 1998

14) Blanco RG, Parithivel VS, Gerst $\mathrm{PH}$, et al : Abdominal wall endometriosis. Am J Surg 185:596-598, 2003

15) Matter M, Schneider N, McKee T: Cystadenocarcinoma of the abdominal wall following Caesarean section: case report and review of the literature. Gynecol Oncol 91:438-443, 2003

16) Bats AS, Zafrani Y, Pautier $P$, et al : Malignant transformation of abdominal wall endometriosis to clear cell carcinoma: case report and review of the literature. Fertil Steril $90: 1197,2008$

17) Ruiz MP, Wallace DL, Connell MT: Transformation of abdominal Wall Endometriosis to Clear Cell Carcinoma. Case Rep Obstet Gynecol 2015 : 123740, 2015

18) Sergent F, Baron M, Marpeau L, et al : Malignant transformation of abdominal wall endometriosis : a new case report. J Gynecol Obstet Biol Reprod (Paris) $35: 186-190,2006$ 